\title{
From Kuo-Brown to today's realistic shell-model calculations
}

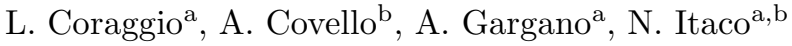 \\ ${ }^{a}$ Istituto Nazionale di Fisica Nucleare, \\ Complesso Universitario di Monte S. Angelo, Via Cintia - I-80126 Napoli, Italy \\ ${ }^{b}$ Dipartimento di Fisica, Università di Napoli Federico II, \\ Complesso Universitario di Monte S. Angelo, Via Cintia - I-80126 Napoli, Italy
}

\begin{abstract}
This paper is an homage to the seminal work of Gerry Brown and Tom Kuo, where shell model calculations were performed for ${ }^{18} \mathrm{O}$ and ${ }^{18} \mathrm{~F}$ using an effective interaction derived from the Hamada-Johnston nucleon-nucleon potential. That work has been the first successful attempt to provide a description of nuclear structure properties starting from the free nucleon-nucleon potential. We shall compare the approach employed in the 1966 paper with the derivation of a modern realistic shell-model interaction for $s d$-shell nuclei, evidencing the progress that has been achieved during the last decades.
\end{abstract}

Keywords: effective interactions, nuclear shell model

PACS: 21.30.Fe, 21.60.Cs, 27.20.+n

\section{Introduction}

The paper by Tom Kuo and Gerry Brown (KB) [1] is a true milestone in the theory of nuclear structure. It has indeed been the first attempt to perform a microscopic nuclear-structure calculation starting from the free nucleon-nucleon $(N N)$ potential which resulted in a quantitative description of the spectroscopy of nuclei belonging to the $s d$ shell.

The KB work was grounded in the general belief, which came out between the end of 1950s and the beginning of 1960s, that a new generation of nuclear structure calculations for finite and infinite systems, based on first principles and free from phenomenological inputs, had to be started. As a matter of fact, $N N$ potentials such as the Yale 2] and the Hamada-Johnston (HJ) ones [3] were able to fit reasonably well the two-nucleon scattering data, both potentials having an infinite short-range repulsion and the one-pion-exchange tail. The handling of the hard-core component of the $N N$ potential in many-body systems was studied by Brueckner and coworkers [4, [5], who introduced an effective potential - the well-known reaction matrix $G$ - which overcomes the singularity at short distances via an infinite sum of particle-particle ladder diagrams. Soon after the Brueckner work, several shell-model calculations were performed, where the 
effective interaction was taken to be the $G$ matrix (see, for instance, [6] and references therein).

A main step forward was then made by Bertsch [7], who studied the role played by the core-polarization diagram corresponding to one-particle-one-hole $(1 p-1 h)$ excitations at second order in perturbation theory. This work evidenced that this diagram, dubbed "bubble", was responsible for a correction to the interaction as large as $30 \%$ of the first-order contribution, when considering the ${ }^{18} \mathrm{O}$ and ${ }^{42} \mathrm{Sc}$ nuclei and using as interaction vertices the $G$-matrix elements derived from the Kallio-Kolltveit potential [8].

Brown and Kuo were well aware that the time was ripe to assemble the new tools, and drew a red line that starting from the free $N N$ interaction ended to the spectroscopic description of a many-nucleon system within a sound theoretical framework. The $s d$-shell effective interaction of [1] was derived starting from the HJ potential, whose hard-core component was renormalized via the calculation of the reaction-matrix $G$. The latter was then employed in the interaction vertices of the perturbative expansion of the effective hamiltonian, and this expansion was performed including terms up to second order.

The KB shell-model effective interaction obtained within this approach was used to calculate the energy spectra of ${ }^{18} \mathrm{O}$ and ${ }^{18} \mathrm{~F}$ yielding good agreement with experiment. This paved the way to a wide sequence of studies, dedicated to both the developement of the perturbative approach to the derivation of the shell-model effective interaction and the assessment of its role in the study of nuclear structure (see for example [9, 10, 11] and references therein).

In this paper, we compare the results obtained in 1966 for $s d$-shell nuclei with those achievable by using a moderm shell-model effective hamiltonian starting from a high-precision $N N$ potential. The aim is to give an idea of the progress made along the line traced by Kuo and Brown more than 50 years ago.

As is well known, during the last decade $N N$ potentials derived within the chiral perturbation theory (ChPT) have provided an approach to the problem of nuclear forces that is well grounded in the quantum cromodynamics. The original idea of deriving realistic two- and three-nucleon forces (2NF and $3 \mathrm{NF}$ ) within the framework of the effective field theory dates back to Weinberg $[12,13$, 14], who considered the most general Lagrangian involving pions and low-energy nucleons consistent with the spontaneously broken chiral symmetry. The shortrange parts of the potential are given in terms of low-energy constants fitted to two-nucleon and, possibly, three-nucleon data.

There are two main advantages in ChPT: the first one is that it generates nuclear two- and many-body forces on equal footing [15, 16, 17]; the second one is that the $N N$ potentials derived within such a framework are naturally tailored for the low-energy regime of the nuclear structure physics. This may allow to avoid the complications of renormalizing the short-range repulsion.

We have constructed a shell-model effective hamiltonian $H_{\text {eff }}$ starting from the so-called $\mathrm{N}^{3} \mathrm{LOW}$ nucleon-nucleon potential [18], a low-momentum potential derived from $\mathrm{ChPT}$ at next-to-next-to-next-to-leading order with a sharp momentum cutoff at $2.1 \mathrm{fm}^{-1}$. The theoretical single-particle (SP) energies and the two-body matrix elements (TBME) of the effective interaction are then ob- 
tained within the framework of the time-dependent degenerate linked-diagram perturbation theory [9], which is an extension of the approach followed in the KB paper.

In the next section we give a few details about the perturbative expansion of the effective shell-model hamiltonian, together with a sketch of the approach followed in the KB work. In Section 3 the results obtained for ${ }^{17,18} \mathrm{O}$ and ${ }^{18} \mathrm{~F}$ with the KB hamiltonian and with that derived from the $\mathrm{N}^{3} \mathrm{LOW}$ potential will be reported and compared with the experimental spectra. Comments and conclusions are drawn in Section 4.

\section{Theoretical framework}

As mentioned in the Introduction, in Ref. [1] the first step in the derivation of the effective interaction was to overcome the difficulty of the short-range singularity due to the hard-core of the $N N$ potential. This problem was tackled calculating the Brueckner reaction matrix $G$ from the HJ potential with the tools available at that time.

As a matter of fact, the calculation of the $G$-matrix was splitted into two subproblems; when dealing with the attractive components of the potential, more precisely the singlet- and triplet-even channels, the $G$-matrix was calculated using the Moszkowski-Scott separation method [19], which is essentially based on dividing the potential into a short-range part $V_{s}$ and a long-range one $V_{l}$.

The separation method cannot be employed when dealing with the components that are repulsive outside the hard-core region, since the essence of this method is that the attractive $V_{s}$ has to balance the short-range repulsion. This is not the case of the singlet- and triplet-odd components of the HJ potential, for which the calculation of the $G$-matrix was carried out using the referencespectrum method [20].

It is worth recalling that during the 1970s more advanced techniques for the calculation of the $G$ matrix were developed. Suffice it to mention here the method proposed by Tsai and Kuo [21], which allows a practically exact calculation of the $G$ matrix and has been largely employed until the early 2000 s. In these years, however, a quite new approach to the renormalization of the $N N$ potential was proposed [22], which consists in constructing a low-momentum $N N$ potential, $V_{\text {low-k }}$, that preserves the physics of $V_{\mathrm{NN}}$ up to a cutoff $\Lambda$. This approach has proved to be an advantageous alternative to the Brueckner Gmatrix method and has become by now a main tool to handle the short-range repulsion of $N N$ potentials like, for instance, those based on the meson theory of nuclear forces. We ourselves have routinely used this approach in several realistic shell-model calculations 23, 24] employing the CD-Bonn $N N$ potential [25].

In the calculations performed in the present work, however, there was no need to renormalize the $N N$ potential. In fact, the $N^{3} \mathrm{LOW}$ potential, while reproducing accurately the experimental deuteron binding energy, low-energy scattering parameters, and phase-shifts of $N N$ scattering up to at least 200 
(a)

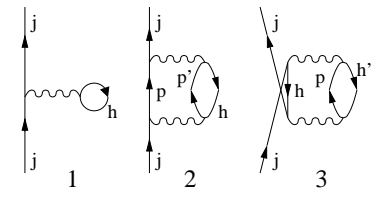

(b)

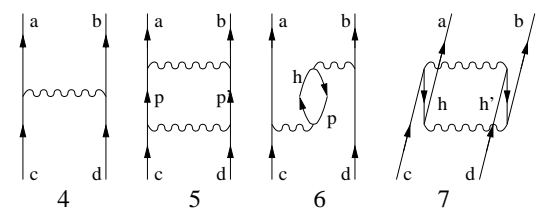

Figure 1: Second-order Goldstone diagrams, with antisymmetrized interaction vertices, included in the KB perturbative expansion of the shell-model hamiltonian. (a) labels the collection of the one-body diagrams, (b) labels the two-body ones. For the sake of simplicity, the "bubble" diagrams differing by exchanges of the valence particle labels are not reported.

$\mathrm{MeV}$ laboratory energy, is a smooth interaction that can be used directly in the derivation of the shell-model hamiltonian.

Starting from this potential, we have derived the shell-model effective hamiltonian within the many-body perturbation theory, as developed by Kuo and coworkers through the 1970s 9, 10]. More precisely, we have used the wellknown $\hat{Q}$-box plus folded-diagram method [26], where the $\hat{Q}$-box is a collection of one- and two-body irreducible valence-linked Goldstone diagrams. Within this framework the effective hamiltonian $H_{\text {eff }}$ can be written in an operator form as

$$
H_{\mathrm{eff}}=\hat{Q}-\hat{Q}^{\prime} \int \hat{Q}+\hat{Q}^{\prime} \int \hat{Q} \int \hat{Q}-\hat{Q}^{\prime} \int \hat{Q} \int \hat{Q} \int \hat{Q}+\ldots,
$$

where the integral sign represents a generalized folding operation, and $\hat{Q}^{\prime}$ is obtained from $\hat{Q}$ by removing first-order terms. In the present calculations the $\hat{Q}$-box includes all diagrams up to third order [27, 28], and the folded-diagram series is summed up to all orders using the Lee-Suzuki iteration method 29]. We sum over the intermediate states between successive vertices whose unperturbed excitation energy is less than $E_{\max }=16 \hbar \omega$, which is sufficiently large to ensure that the matrix elements of the effective hamiltonian are almost independent from the value of $E_{\max }$.

The effective hamiltonian so obtained contains one- and two-body terms, but we use a subtraction procedure so that only the two-body term is retained while the SP energies are taken from experiment. In particular, in the calculations of ${ }^{18} \mathrm{O}$ and ${ }^{18} \mathrm{~F}$ presented here we consider ${ }^{16} \mathrm{O}$ as closed core and take the two SP energy spacings of the $s d$ space from the experimental spectrum of ${ }^{17} \mathrm{O}[30$ ] while the absolute energies are determined from the experimental binding energies of ${ }^{17} \mathrm{O}$ and ${ }^{17} \mathrm{~F}$ with respect to ${ }^{16} \mathrm{O}[31]$, as was done in the $\mathrm{KB}$ paper. The two-body term employed in the latter was instead calculated considering only corrections to the $G$ matrix as arising from $2 \hbar \omega 1 p-1 h$ excitations 
of the ${ }^{16} \mathrm{O}$ core. Within our procedure, this corresponds to include a unique second-order diagram, the "bubble" diagram 6 Fig. 1 $\mathrm{b}$, in the calculation of the $\hat{Q}$-box, without performing the folded-diagram expansion. It is worth noting that the latter is necessary to remove violations of time ordering coming from the factorization of diagrams from second order on. Actually, corrections other than the $1 p-1 h$ ones, corresponding to diagrams 5 and 7 of Fig. 1 b, were also discussed in [1] but not explicitly included in the calculations. It is also worth noting that the number of intermediate states taken into account when calculating the bubble diagram was not enough to achieve a satisfactory convergence, as was later pointed out in the papers by Vary et al. [32], Kung et al. 33], and Sommermann et al. 34].

In the following section, the spectra for ${ }^{18} \mathrm{O}$ and ${ }^{18} \mathrm{~F}$ obtained with the $\mathrm{KB}$ and $N^{3} \mathrm{LOW}$ effective interactions are compared with experiment. For the sake of completeness, we also compare our calculated SP energies for ${ }^{17} \mathrm{O}$, obtained consistently with the theory described above, with those of the KB paper resulting from inclusion of the three one-body first and second-order diagrams shown in Fig. 17.

\section{Results}

Let us start with the spectrum of ${ }^{18} \mathrm{O}$. In Fig. 2 the experimental low-energy spectrum referred to the ${ }^{16} \mathrm{O}$ ground-state energy is compared with those obtained from the KB and $N^{3} \mathrm{LOW}$ effective interactions. Note that the KB spectrun is scaled by twice the experimental ${ }^{17} \mathrm{O}$ ground-state energy with respect to that reported in [1]. In the $\mathrm{KB}$ work, it is clearly shown that the inclusion of $1 p-1 h$ corrections to the $G$ matrix interaction leads to a lowering of the low-lying states and a raising of the higher ones, providing a substantial improvement in the description of ${ }^{18} \mathrm{O}$. As a matter of fact, as we see from Fig. 2, the agreement of the KB results with experiment is quite good. The binding energy is overestimated by only about $300 \mathrm{keV}$, and the differences between the observed and calculated spacings do not go beyond $500 \mathrm{keV}$. The $N^{3} \mathrm{LOW}$ calculations, when excluding the second $0^{+}$state, lead to a further improvement. In particular, the energies of the yrast $2^{+}$and $4^{+}$states come very close to the experimental values.

The energy of the $0_{2}^{+}$state is largely overestimated by our calculation. Our predicted level lies about $1.2 \mathrm{MeV}$ above the experimental one, which may be traced to the fact that this state contain a relevant collective $4 p-2 h$ component as testified by the large experimental value of the $B\left(E 2 ; 0_{2}^{+} \rightarrow 2_{1}^{+}\right)=17 \pm 2$ W.u. [30]. It is indeed suprising that the discrepancy reduces to $700 \mathrm{keV}$ in the KB case, where no $2 p-2 h$ corrections to the $G$ matrix were included.

We now come to discuss the results for ${ }^{18} \mathrm{~F}$, which provides a direct test of the proton-neutron interaction. In Fig. 3 , the two calculated spectra are compared with the experimental one. As for the ${ }^{18} \mathrm{O}$ case, the $\mathrm{KB}$ spectrum shown in the figure is scaled with respect to the original one by using the experimental ground-state energies of the two one-valence-particle nuclei. 


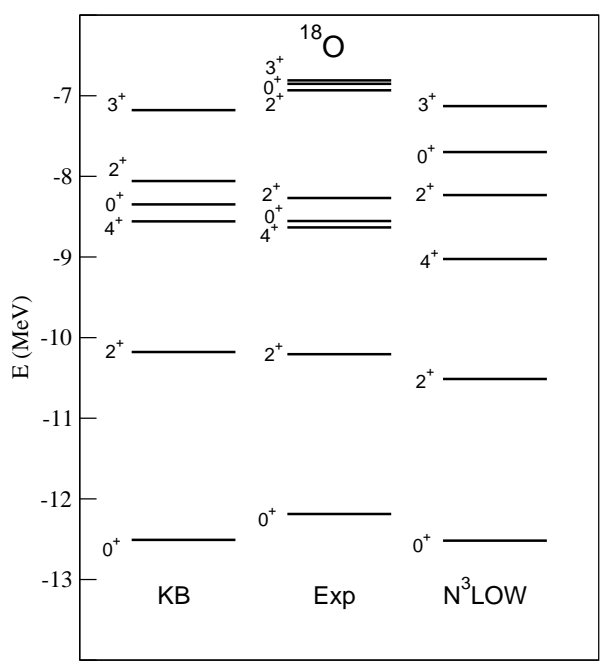

Figure 2: Experimental [30] and calculated low-energy spectra for ${ }^{18} \mathrm{O}$ (see text for details).

We see that, although the energy differences between experiment and theory for both calculations are quite similar to those found for ${ }^{18} \mathrm{O}$, the $\mathrm{KB}$ spectrum, at variance with the $\mathrm{N}^{3} \mathrm{LOW}$ one, does not give the correct sequence of the low-lying $T=0,1$ states. As concerns the effects of including $2 p$ and $2 p-2 h$ excitations in the derivation of the effective interaction, no definite conclusions were drawn in the KB paper owing to possible-double counting or convergence problems related to the adopted scheme.

Finally, in Table 1 we compare the SP energy spacings obtained with KB and $\mathrm{N}^{3} \mathrm{LOW}$ interactions with the experimental ones between the $J^{\pi}=\frac{5}{2}^{+}, \frac{1}{2}^{+}, \frac{3}{2}^{+}$ states that have the largest spectroscopic factors in the ${ }^{16} \mathrm{O}(\mathrm{d}, \mathrm{p}){ }^{17} \mathrm{O}$ transfer reaction [30].

\begin{tabular}{|c|l|l|l|}
\hline$J^{\pi}$ & $\mathrm{KB}$ & $\mathrm{N}^{3} \mathrm{LOW}$ & Expt. \\
\hline \hline$\frac{5}{2}^{+}$ & $0.0(-2.64)$ & $0.0(-3.211)$ & $0.0(-4.144)$ \\
$\frac{1}{2}^{+}$ & 0.11 & 0.835 & 0.871 \\
$\frac{3}{2}^{+}$ & 5.51 & 6.281 & 5.085 \\
\hline
\end{tabular}

Table 1: Experimental and theoretical SP energy spacings of ${ }^{17} \mathrm{O}$ obtained with $\mathrm{KB}$ and $\mathrm{N}^{3} \mathrm{LOW}$ potentials (see text for details). The ground-state energies with respect to ${ }^{16} \mathrm{O}$ are reported in parenthesis.

We see that both calculations reproduce reasonably well the observed SP energy spacings. More precisely, the $\epsilon_{1 / 2^{+}}-\epsilon_{5 / 2^{+}}$spacing calculated with $\mathrm{N}^{3} \mathrm{LOW}$ comes closer to the experimental value while the KB interaction yields a spinorbit splitting $\epsilon_{3 / 2^{+}}-\epsilon_{5 / 2^{+}}$in better agreement with experiment. The larger 


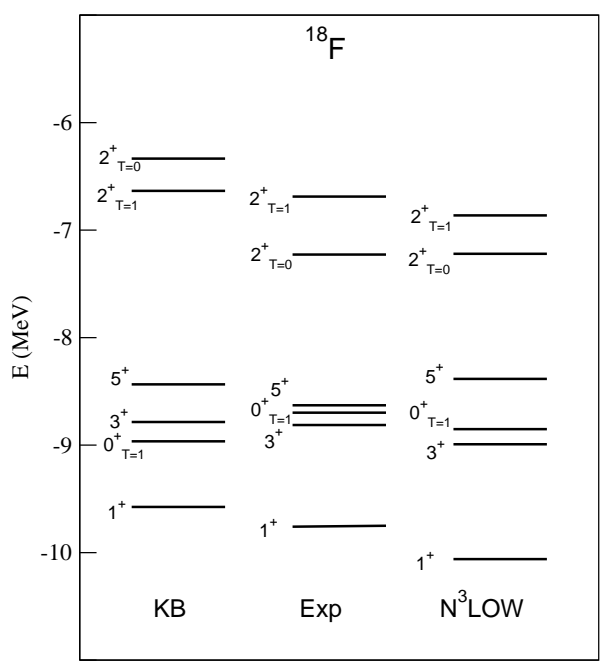

Figure 3: Experimental [30] and calculated low-energy spectra for ${ }^{18} \mathrm{~F}$ (see text for details).

value of the spin-orbit splitting obtained with the $\mathrm{N}^{3} \mathrm{LOW}$ calculation can be ascribed to the contribution to the perturbative expansion coming from thirdorder diagrams. As a matter of fact, we have verified that, starting from the $\mathrm{N}^{3} \mathrm{LOW}$ potential and using the folded-diagram expansion with a $\hat{Q}$-box up to second order, namely by including only the three one-body diagrams of Fig. 1a, we obtain $\epsilon_{1 / 2^{+}}-\epsilon_{5 / 2^{+}}=0.626 \mathrm{MeV}$ and $\epsilon_{3 / 2^{+}}-\epsilon_{5 / 2^{+}}=4.945 \mathrm{MeV}$, the latter value being very close to the $\mathrm{KB}$ one shown in Table 1 . As regards the binding energies, the values obtained from the $\mathrm{N}^{3} \mathrm{LOW}$ and $\mathrm{KB}$ calculations are both within acceptable limits, the former being only about $900 \mathrm{keV}$ smaller than the experimental value.

\section{Summary and conclusions}

In this work, we have revisited the approach to realistic shell-model calculations followed by Gerry Brown and Tom Kuo in their pioneering paper [1], where the Hamada-Johnston potential was employed to study the spectroscopic properties of ${ }^{18} \mathrm{O}$ and ${ }^{18} \mathrm{~F}$. The KB paper represents a breakthrough in the history of nuclear structure, since it showed for the first time that it was possible to reach a reasonable degree of accuracy in the description of many-nucleon systems starting from the free $N N$ potential.

For the sake of comparison, we have constructed a modern realistic shellmodel hamiltonian and performed calculations for the same nuclei ${ }^{18} \mathrm{O}$ and ${ }^{18} \mathrm{~F}$. We have employed the chiral $\mathrm{N}^{3} \mathrm{LOW} N N$ potential within the framework of the $\hat{Q}$-box plus folded-diagram method, which is substantially an upgrade of the perturbative expansion carried out in the KB paper. 
As we have already pointed out in Section 2, the shell-model calculations employing realistic effective interactions have by now entered the mainstream of nuclear structure theory, having proved to be able to provide an accurate description of the spectroscopic properties of nuclei in different mass regions (see, for instance, Ref. [11, 23, 35] and references therein). However, in this contribution to the Gerry's memorial volume, we deemed it appropriate to only focus on the two nuclei studied in the KB paper, as the starting point of a new generation of nuclear structure calculations.

From the results presented in Section 3 it appears that the modern calculations lead on the whole to a moderately improved description of the experimental data with respect to the KB results. This shows the substantial soundness of the original KB approach, which was able to catch the main aspects of the physics of many-nucleon systems within the framework of the shell model. However, as we have discussed in Section 2, the framework for realistic shell-model calculations has substantially improved over the initial one and rests now on solid theoretical foundations, as regards both the starting $N N$ potential and the many-body technique for constructing the effective interaction. It is indeed very gratifying

that the long journey initiated by Gerry Brown and Tom Kuo has been crowned with success.

\section{References}

\section{References}

[1] T. T. S. Kuo, G. E. Brown, Nucl. Phys. 85 (1966) 40.

[2] K. E. Lassila, J. M. H. Hull, H. M. Ruppel, F. A. McDonald, G. Breit 126 (1962) 881.

[3] T. Hamada, I. D. Johnston, Nucl. Phys 34 (1962) 382.

[4] K. A. Brueckner, C. A. Levinson, H. M. Mahmoud, Phys. Rev. 95 (1954) 217.

[5] K. A. Brueckner, C. A. Levinson, Phys. Rev. 97 (1955) 1344.

[6] A. Kahana, H. C. Lee, K. Scott, Phys. Rev. 185 (1969) 1378.

[7] G. F. Bertsch, Nucl. Phys. 74 (1965) 234.

[8] A. Kallio, K. Kolltveit, Nucl. Phys. 53 (1964) 87.

[9] T. T. S. Kuo, E. Osnes, Lecture Notes in Physics, vol. 364, Springer-Verlag, Berlin, 1990.

[10] M. Hjorth-Jensen, T. T. S. Kuo, E. Osnes, Phys. Rep. 261 (1995) 125.

[11] L. Coraggio, A. Covello, A. Gargano, N. Itaco, T. T. S. Kuo, Prog. Part. Nucl. Phys. 62 (2009) 135. 
[12] S. Weinberg, Physica 96A (1979) 327.

[13] S. Weinberg, Phys. Lett. B 251 (1990) 288.

[14] S. Weinberg, Nucl. Phys. B 363 (1991) 3.

[15] S. Weinberg, Phys. Lett. B 295 (1992) 114.

[16] U. van Kolck, Phys. Rev. C 49 (1994) 2932.

[17] R. Machleidt, D. R. Entem, Phys. Rep. 503 (2011) 1.

[18] L. Coraggio, A. Covello, A. Gargano, N. Itaco, T. T. S. Kuo, D. R. Entem, R. Machleidt, Phys. Rev. C 75 (2007) 024311.

[19] S. A. Moszkowski, B. L. Scott, Ann. Phys (N.Y.) 11 (1960) 65.

[20] H. A. Bethe, B. H. Brandow, A. G. Petscheck, Phys. Rev. 129 (1963) 225.

[21] S. F. Tsai, T. T. S. Kuo, Phys. Lett. B 39 (1972) 427.

[22] S. Bogner, T. T. S. Kuo, L. Coraggio, A. Covello, N. Itaco, Phys. Rev. C 65 (2002) 051301(R).

[23] L. Coraggio, A. Covello, A. Gargano, N. Itaco, Phys. Rev. C 80 (2) (2009) 021305 .

[24] L. Coraggio, A. Covello, A. Gargano, N. Itaco, Phys. Rev. C 88 (2013) 041304.

[25] R. Machleidt, Phys. Rev. C 63 (2001) 024001.

[26] T. T. S. Kuo, S. Y. Lee, K. F. Ratcliff, Nucl. Phys. A 176 (1971) 65.

[27] L. Coraggio, A. Covello, A. Gargano, N. Itaco, Phys. Rev. C 81 (6) (2010) 064303.

[28] L. Coraggio, A. Covello, A. Gargano, N. Itaco, T. T. S. Kuo, Ann. Phys. 327 (2012) 2125.

[29] K. Suzuki, S. Y. Lee, Prog. Theor. Phys. 64 (1980) 2091.

[30] Data extracted using the NNDC On-line Data Service from the ENSDF database, file revised as of September 12, 2013.

[31] M. Wang, G. Audi, A. H. Wapstra, F. Kondev, M. MacCormick, X. Xu, B. Pfeiffer, Chinese Phys. C 36 (2012) 1603.

[32] J. P. Vary, P. U. Sauer, C. W. Wong, Phys. Rev. C 7 (1973) 1776.

[33] C. L. Kung, T. T. S. Kuo, K. F. Ratcliff, Phys. Rev. C 19 (1979) 1063.

[34] H. M. Sommermann, H. Müther, K. C. Tam, T. T. S. Kuo, A. Faessler, Phys. Rev. C 23 (1981) 1765.

[35] L. Coraggio, A. Covello, A. Gargano, N. Itaco, Phys. Rev. C 89 (2014) 024319 . 